\title{
The Efficacy of On-Site Evaluation for Identification of Transplant Pancreas
}

\author{
Natasha Berg ${ }^{a}$ Jim Gehl ${ }^{b}$ Mark Vande Haar ${ }^{a}$ Michael Balco $^{a} \quad$ Peter Kulesza ${ }^{a}$ \\ Departments of a Pathology and ${ }^{b}$ Radiology, Feinberg School of Medicine, Northwestern University, Chicago, III., USA
}

\section{Key Words \\ Pancreas transplant · Fine needle aspiration . \\ Cytopathology · Rejection · On-site evaluation}

\begin{abstract}
Objective: Pancreatic transplantation has been relatively uncommon until the last 2 decades, but is now an accepted therapeutic intervention for type I diabetes. Core biopsies of pancreas transplants are frequently performed to rule out rejection, which is a leading cause of graft failure. Often, it is difficult to localize the graft for biopsy by imaging. Our objective was to determine whether on-site assessment by cytopathology can assist in procedure protocols for biopsy of pancreas transplants. Study Design: We reviewed cytology and surgical pathology reports from 68 instances of pancreas transplant biopsy at our institution and evaluated the correlation between cytology (fine needle aspiration, touch preparation or both) and the final core biopsy. Results: Pancreatic tissue was identified by on-site assessment in $56 / 68$ cases and was present on core biopsy in 53/68 cases. There were 3 cases where fine needle aspiration/touch preparation results were not concordant with final core biopsy results. The positive predictive value of a positive on-site assessment was $96.4 \%$. More importantly, the negative predictive value of on-site assessment was $100 \%$. Conclusions: On-site evaluation by cytopathology is highly effective for the identification of transplant pancreas. In cases where no pancreatic tissue is identified by on-site assessment, relocalization of the biopsy needle is recommended.

(c) 2013 S. Karger AG, Basel
\end{abstract}

\section{Introduction}

Pancreatic transplantation was first performed at the University of Minnesota in 1966, and has been increasingly accepted as a therapeutic option for patients with type I diabetes. Outcomes for patients include independence from insulin, prevention and even reversal of some diabetic sequelae, including neuropathy and nephropathy. Surgical techniques and graft procurement have evolved steadily to the point where grafts may survive successfully for up to 20 years $[1,2]$.

Allografts may be from living or cadaveric donors and are sometimes divided between 2 recipients. Donor vasculature, including the superior mesenteric, splenic artery, and common, internal and external iliac arteries, is harvested with the allograft along with a donor duodenal stump containing the ampulla of Vater. Currently, exocrine drainage of the transplant is most commonly achieved by anastomosis of the duodenal stump with recipient small bowel. The graft is typically located in the lower abdomen to the right of the midline, with cranial and caudal orientation determined by the route of venous drainage. Alternatively, the duodenal stump may be anastomosed to the superior aspect of the recipient bladder to provide exocrine bladder drainage $[1,3]$.

After transplant, patients are monitored for pancreatic function and evidence of rejection. If laboratory values are

This research was presented in poster format at the 60th annual scientific meeting of the American Society of Cytopathology.

\section{KARGER}

E-Mail karger@karger.com

www.karger.com/acy
C 2013 S. Karger AG, Basel

0001-5547/13/0575-0443\$38.00/0
Correspondence to: Dr. Peter Kulesza

Department of Pathology, Northwestern Memorial Hospital

303 East Chicago Avenue, Ward 3-140 W127

Chicago, IL 60611 (USA)

E-Mail p-kulesza@ northwestern.edu 
suggestive of rejection, a core biopsy is performed to evaluate the transplant organ $[4,5]$. In the early days of pancreatic transplantation a variety of biopsy methods were available, including cystoscopic and open methods. However, since the introduction of percutaneous biopsy in the 1990s, this has become the preferred method of sampling, usually guided by ultrasound (US) or computed tomography (CT) or both. Due to advances in surgical practice, individual physiological variations, vascular supply, duct drainage and presence/absence of concurrent renal transplant, the location and placement of pancreatic allografts may vary. Sonographically, the normal transplant pancreas is uniformly hypoechoic relative to the surrounding mesenteric and omental fat. US is often the first choice for image-guided biopsy given its real-time capability, low cost and lack of ionizing radiation $[1,5]$.

After locating a suitable sonographic window with grayscale US, vascular structures are interrogated with Doppler US. Challenges of US-guided biopsy include the inability to obtain a suitable sonographic window, principally secondary to the overlying bowel. Large body habitus, small size of the allograft and deep location of the transplant can present additional challenges [6]. After anesthetizing subcutaneous and deep abdominal wall layers with lidocaine, the biopsy device is advanced into either the pancreatic head or tail, avoiding major vascular structures, under real-time sonographic guidance. In a series of 426 US-guided biopsies using this technique, $88 \%$ of specimens were adequate for analysis, with bleeding complications occurring in less than $2 \%[7,8]$.

Although US-guided biopsy is preferred for its realtime capability, CT-guided biopsy can be pursued when a suitable sonographic window is not available. Oral contrast may be administered approximately $2 \mathrm{~h}$ prior to attempted biopsy, although the duodenal stump does not reliably fill with oral contrast. In the absence of oral contrast, the homogenous pancreatic transplant can be similar in appearance to the nondistended adjacent small bowel. The overlying bowel can also be present in CT-guided biopsies. In such cases, repositioning the patient or the use of compression bands may be used to displace bowel loops $[3,7]$.

For the reasons described above, the radiological appearance of the transplanted tissue is frequently indistinct, and therefore it may not be possible to accurately localize the graft using either US or CT. Thus, the standard of practice is evolving to include fine needle aspiration (FNA) or touch preparation (TP) of the core which directs the core biopsy needle to the correct location. This is performed as on-site assessment by cytopathologists for the presence of pancreatic elements, including ductal or acinar cells. Communication of these results to the interventional radiologist allows for a more directed procedure and an optimal core specimen.

We retrospectively evaluated the correlation between FNA and TP reports and the final core biopsy reports for the presence of pancreatic tissue to evaluate the efficacy of on-site assessment as a method for identification of transplant pancreas.

\section{Materials and Methods}

Institutional Review Board approval was obtained before the initiation of the study. Through a retrospective records search, we identified 68 instances of pancreas transplant biopsy that occurred at our institution between January 2006 and February 2012. All of the transplants were performed for the treatment of type I diabetes and the length of time from transplant to biopsy varied from less than 1 month to 9 years. Patient age ranged from 21 to 71 years. In each case, the goal of the core biopsy was to assess the graft for evidence of rejection.

All biopsies were percutaneous and were obtained under US or CT guidance. FNAs were obtained with a 25-gauge needle and core biopsies were obtained with a 20 -gauge core needle. In each case, an on-site evaluation was performed either by FNA directly prior to core biopsy or by TP of the obtained core biopsy or a combination of both. Slides were stained with Diff-Quik and examined on site by a cytopathologist to assess for the presence of pancreatic tissue. In all cases with both an FNA and a core biopsy with TP, separate charges were billed for the FNA, TP and core. Separate charges were billed for on-site evaluations and final diagnoses.

Cores were fixed in 10\% neutral-buffered formalin and sent for processing in surgical pathology. Rejection of transplant pancreas is assessed at our institution using the Banff schema for pancreas allograft rejection [9].

Cytopathology and surgical pathology reports were obtained for all cases and reviewed for the presence or absence of pancreatic tissue. In those cases where there was a discrepancy between FNA or TP and the final core biopsy report, the corresponding slides from both were reviewed by a pathologist. Correlation was assessed by determining the positive and negative predictive values of FNA/TP and core biopsy results.

\section{Results}

Pancreatic tissue was identified by on-site assessment of FNA, TP or a combination of both. Of 68 cases, 47 used TP only for assessment, 12 used FNA, and 9 used a combination of both. In the cases with cores, the number of cores obtained ranged from 1 to 5 , with an average of 2.09 . In the FNA cases, the number of passes ranged from 1 to 4 with an average of 1.63. Of the 9 cases where both FNA and TP were performed, 4 found that both methods provided diagnostic material, 1 had a nondiagnostic TP portion, and 4 had nondiagnostic FNA. 

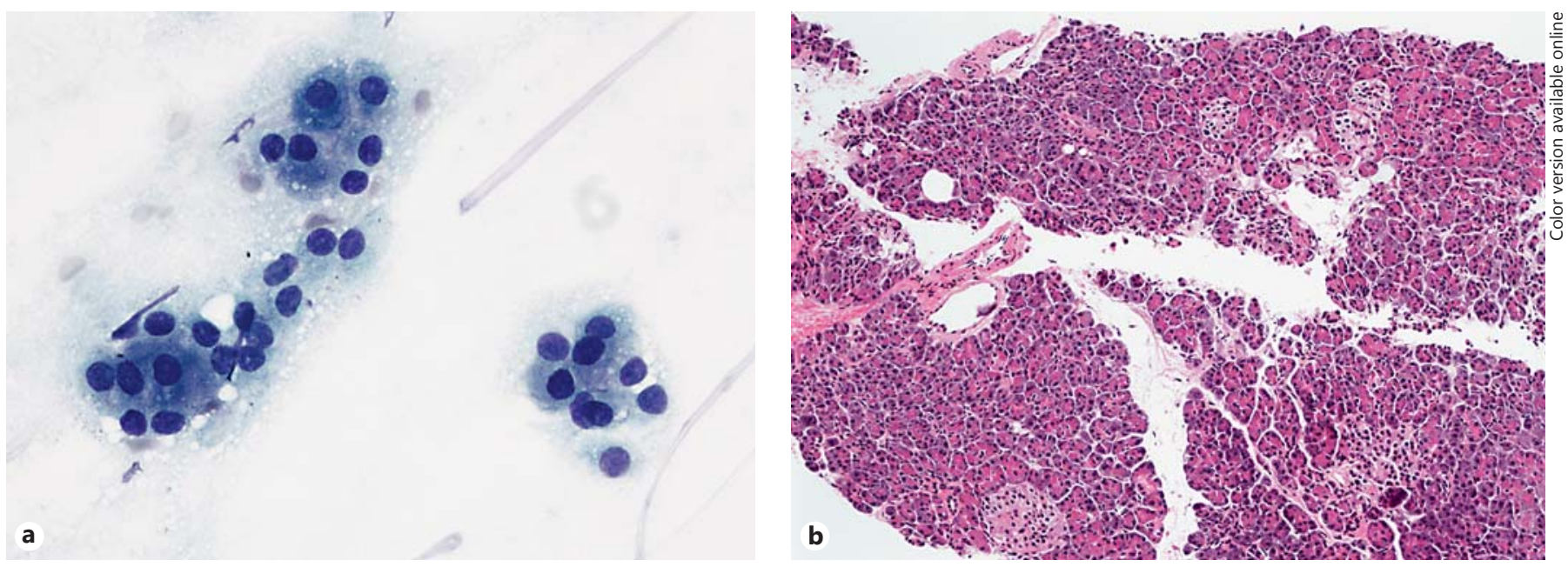

Fig. 1. Case with concordant TP and tissue biopsy. a Cytology TP demonstrates pancreatic acinar cells. b Surgical pathology core demonstrates pancreatic tissue with no evidence of rejection. a Diff-Quik. $\times 400$. b HE. $\times 100$.
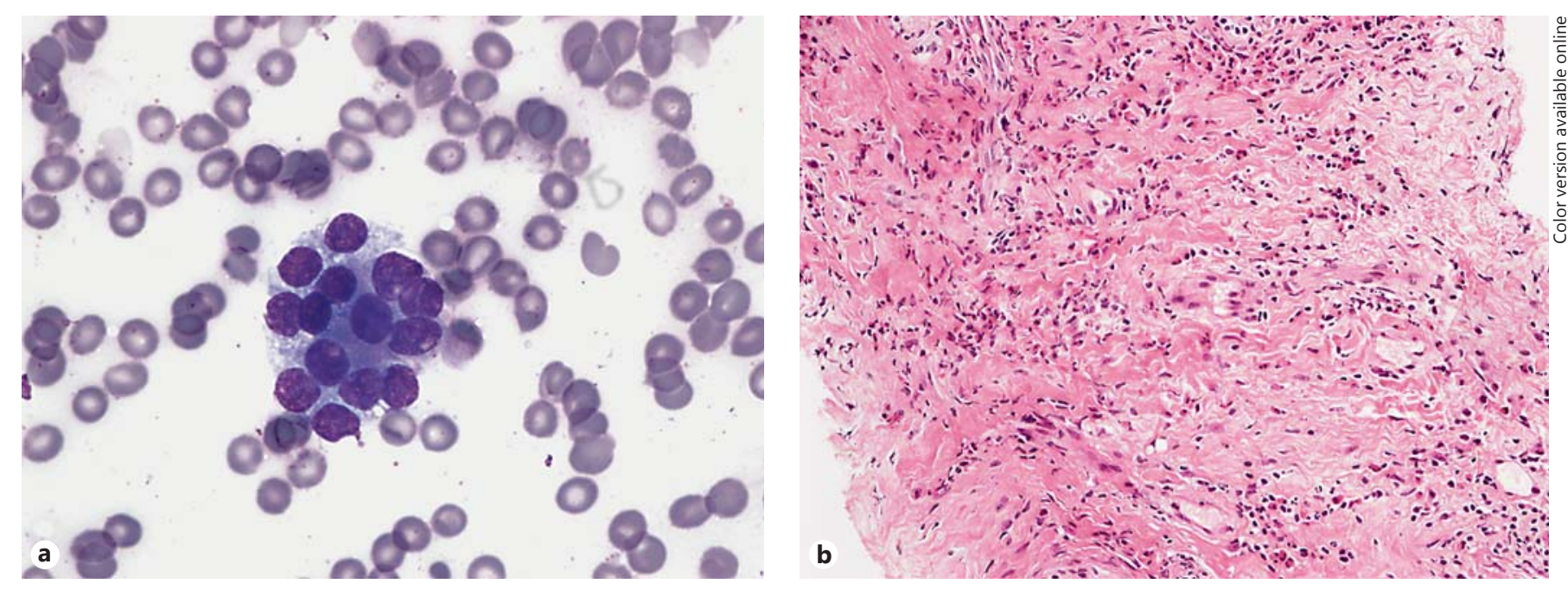

Fig. 2. Case with discordant TP and tissue biopsy. a Cytology TP demonstrates pancreatic acinar cells. b Surgical pathology core demonstrates fibrous tissue with inflammation. a Diff-Quik. ×400. b HE. ×200.

On-site assessment identified pancreatic elements in $56 / 68$ cases (fig. 1a, 2a). Core biopsies were found to contain pancreatic tissue in 53/68 cases (table 1 ). The positive predictive value of a positive on-site assessment was $96.4 \%$. The negative predictive value of a negative on-site assessment was $100 \%$.

Rejection was detected in 23 out of the 53 cores that were found to contain pancreatic tissue (43.4\%). The remaining successful cores demonstrated pancreatic tissue without rejection (fig. 1b). Cores that did not contain pancreatic tissue contained a variety of other elements includ- ing fibromuscular tissue, fibrous tissue and nerve, duodenum, and fibrous tissue with giant cell reaction (fig. $2 \mathrm{~b}$ ).

There were only 3 cases where FNA/TP results were not concordant with final core biopsy results. Of these cases, 1 was TP only, 1 was FNA only, and 1 was a combination of TP and FNA. In each case, the slides were reviewed by a pathologist and the presence or absence of diagnostic tissue was verified. In all 3 discrepant cases, the cytopathology slides demonstrated acinar or ductal cells (fig. 2a), but no pancreatic tissue was obtained on final core biopsy. 
Table 1. Pancreas transplant biopsies: cytology and surgical pathology $(n=68)$

\begin{tabular}{lllr}
\hline $\begin{array}{c}\text { Cytology - positive cases } \\
\text { (pancreatic tissue identified) }\end{array}$ & 56 & $\begin{array}{l}\text { Core positive } \\
\text { Core negative }\end{array}$ & $\begin{array}{r}53 \\
3\end{array}$ \\
\hline $\begin{array}{c}\text { Cytology - negative cases } \\
\text { (no pancreatic tissue identified) }\end{array}$ & 12 & $\begin{array}{l}\text { Core positive } \\
\text { Core negative }\end{array}$ & 0 \\
12
\end{tabular}

\section{Discussion}

The results demonstrate that on-site assessment of an FNA or TP is efficacious in identifying transplant pancreas (with a $96 \%$ positive predictive value) and may be considered a necessary step for localization of the pancreas prior to transplant biopsy. In previous studies, success rates for US- and CT-guided biopsies of pancreas transplants have been $83 \%$ [4] and 85\% [5]. We hypothesize that this number could approach $100 \%$, based on dialogue between the cytopathologist and interventional radiologist.

Our study used a mixture of FNA and TP for the onsite assessment. In cases where both were available for evaluation, the FNA was more likely to be nondiagnostic. However, FNA was still demonstrated to be a valuable modality for identifying the graft when used alone; 11 of the 12 cases where only FNA was used for assessment showed correlation with the final core biopsy. For this reason, we consider either FNA or TP a viable option for on-site assessment in biopsy of transplant pancreas.
A previous study has suggested the possibility of diagnosing rejection of transplant pancreas on FNA [10], although the authors acknowledged that they had a limited sample to evaluate. FNA offers the theoretical advantage of being able to sample multiple areas of the transplant graft; however, it is difficult to identify subtle features such as arteritis on smear preparations. Based on our experience with this series, we feel that it would be difficult to confirm with certainty the presence of rejection and accurately distinguish it from other entities, including posttransplant ischemic pancreatitis, CMV pancreatitis, bacterial or fungal infection, peripancreatitis, or recurrent autoimmune disease [9].

Given the pattern of transplant procedures to utilize less donor tissue and to place the tissue in a deeper location, we expect the need for cytopathology-guided biopsy to increase. The results presented here assist in guiding procedure protocols: in cases where no pancreatic tissue is obtained on FNA or TP made from the core biopsy, relocation of the biopsy needle is advisable.

The negative predictive value of on-site assessment by these methods was determined to be $100 \%$; in no cases where the FNA or TP were negative was pancreatic tissue obtained on core biopsy. Given the high negative predictive value of TP or FNA in situations where no pancreatic tissue is identified by these methods, it is advisable to attempt relocation and reassessment, since it is highly unlikely that the core biopsy will yield pancreatic tissue. Our data suggest that biopsy of the transplant pancreas with on-site assessment by FNA or TP is effective and appropriate for routine clinical practice.

\section{References}

-1 Sutherland DE, Gruessner RW, Dunn DL, Matas AJ, Humar A, Kandaswamy R, Mauer SM, Kennedy WR, Goetz FC, Robertson RP, Gruessner AC, Najarian JS: Lessons learned from more than 1,000 pancreas transplants at a single institution. Ann Surg 2001;233:463501.

-2 Gruessner AC, Sutherland DE: Pancreas transplant outcomes for United States cases as reported to the United Network for Organ Sharing (UNOS) and the International Pancreas Transplant Registry (IPTR). Clin Transpl 2008:45-56.

$\checkmark 3$ Vandermeer FQ, Manning MA, Frazier AA, Wong-You-Cheong JJ: Imaging of wholeorgan pancreas transplants. Radiographics 2012;32:411-435.

$\checkmark 4$ Lee BC, McGahan JP, Perez RV, Boone JM: The role of percutaneous biopsy in detection of pancreatic transplant rejection. Clin Transplant 2000;14:493-498.

5 Malek SK, Potdar S, Martin JA, Tublin M, Shapiro R, Fung JJ: Percutaneous ultrasoundguided pancreas allograft biopsy: a singlecenter experience. Transplant Proc 2005;37: 4436-4437.

6 Nikolaidis P, Amin RS, Hwang CM, et al: Role of sonography in pancreatic transplantation. Radiographics 2003;23:939-949.

$>7$ Daly B, O’Kelly K, Klassen D: Interventional procedures in whole organ and islet cell pancreas transplantation. Semin Intervent Radiol 2004;21:335-343.

$>8$ Klassen DK, Weir MR, Cangro CB, Bartlett ST, Papadimitriou JC, Drachenberg CB: Pancreas allograft biopsy: safety of percutaneous biopsy - results of a large experience. Transplantation 2002;73:553-555.
Drachenberg CB, Odorico J, Demetris AJ, Arend L, Bajema IM, Bruijn JA, Cantarovich D, Cathro HP, Chapman J, Dimosthenous $\mathrm{K}$, Fyfe-Kirschner B, Gaber L, Gaber O, Goldberg J, Honsová E, Iskandar SS, Klassen DK, Nankivell B, Papadimitriou JC, Racusen LC, Randhawa P, Reinholt FP, Renaudin K, Revelo PP, Ruiz P, Torrealba JR, VazquezMartul E, Voska L, Stratta R, Bartlett ST, Sutherland DE: Banff schema for grading pancreas allograft rejection: working proposal by a multi-disciplinary international consensus panel. Am J Transplant 2008;8: 1237-1249.

10 Sariya D, Kluskens L, Assad L, Treaba D, Reddy V, Gattuso P: Diagnostic role of fine-needle aspiration of pancreatic allograft to detect rejection. Diagn Cytopathol 2002;27:266270 